\title{
AS NOVAS TENDÊNCIAS NA EDUCAÇÃO E NO USO DE TECNOLOGIAS DA INFORMÁTICA PARA IDOSOS
}

\author{
Arlete Eli Kunz da Costa ${ }^{1}$; Noeli Juarez Ferla ${ }^{2}$; Luis Felipe Pissaia ${ }^{3}$; Claudete Moreschi ${ }^{4}$
}

\begin{abstract}
Resumo: $\mathrm{O}$ presente estudo objetivou conhecer as novas tendências na área da educação e no uso de tecnologias da informática em idosos do Vale do Taquari/RS, Brasil. Caracteriza-se como uma pesquisa de campo, exploratória e descritiva, com abordagem qualitativa, desenvolvida com 75 idosos. A idade dos participantes variou entre os 60 a 89 anos, sendo 52 mulheres e 23 homens. A maioria dos idosos carecem de apoio educacional no ensino escolar básico. Os idosos referem a importância do uso de tecnologias da informática como meio de comunicação. Todavia, a informática, bem como outras tecnologias foram incorporadas ao meio social recentemente e nem sempre os idosos sabem manusear estes aparelhos eletrônicos. $\mathrm{O}$ idosos mencionam algumas dificuldades econômicas em relação ao acesso à educação e tecnologia. Emergiu também que os idosos necessitam de uma boa saúde a fim de usufruir de algumas atividades que não dispunham quando jovens e hoje na terceira idade possuem esta oportunidade. Para os idosos, possuir saúde é estar bem, não sentir dor e realizar suas tarefas diárias como desejam. Conclui-se que as equipes multiprofissionais possuem o dever de instrumentalizar a comunidade sobre o envelhecimento, capacitando às famílias e responsabilizando os indivíduos nos processos de envelhecimento seguro e saudável.
\end{abstract}

Palavras-chave: Longevidade. Centros Comunitários para Idosos. Informática em Saúde.

Abstract: This study aimed to identify the new trends in education and the use of information technologies in elderly Vale do Taquari/RS, Brasil. It is characterized as a field research, exploratory and descriptive, with a qualitative approach, developed with 75 seniors. The age of participants ranged from 60 to 89 years, 52 women and 23 men. Most seniors lack of educational support in primary school education. The elderly refer to the importance of the use of information technologies as a means of communication. However, information technology and other technologies have been incorporated into the recently social environment and not always know the elderly handle these electronic devices. The elderly mention some economic difficulties in relation to access to education and technology. Also emerged that the elderly need good health to enjoy some activities that they had when they were young and now the elderly have this opportunity. For the elderly, have health is to be good, no pain and perform their daily tasks as they wish. We conclude that multidisciplinary teams have a duty to equip the community on aging, empowering families and responsible individuals in safe and healthy aging processes.

Keywords: Longevity. Senior Centers. Health Informatics.

1 Enfermeira. Doutora em Ambiente e Desenvolvimento. Docente do Centro de Ciências Biológicas e da Saúde. Centro Universitário UNIVATES. arlete.costa@univates.br

2 Biólogo. Doutor em Ciências. Docente do Centro Universitário UNIVATES.njferla@univates.br

3 Enfermeiro. Mestrando em Ensino. Centro Universitário UNIVATES. 1pissaia@universo.univates.br

4 Enfermeira. Doutora em Ambiente e Desenvolvimento. Centro Universitário UNIVATES. clau_moreschi@yahoo.com.br 


\section{INTRODUÇÃO}

O crescimento da população idosa pode ser observado em vários países, no entanto, nos últimos anos os percentuais superaram as estimativas, este efeito está relacionado com drástica queda nas taxas de natalidade e consequente aumento da expectativa de vida, devido à melhoria nos fatores socioculturais do século XXI (MARIN; PANES, 2015). No Brasil, os idosos representam 10\% da população total, com perspectiva de chegar a 25 milhões de pessoas em 2015 e em 2050 o número de idosos dobre e atinja $19 \%$ da população brasileira (IBGE, 2008).

Apesar de o crescimento ser visivelmente gradual e natural nas sociedades em processo de desenvolvimento, em nosso país, as altas taxas causam desconforto devido à falta de apoio de políticas públicas assistenciais a terceira idade (COSTA et al., 2015). Os direitos a saúde da população foram definidos por meio da Constituição Federal de 1988, estimulando a criação do Sistema Único de Saúde (SUS), que foi implementado pela Lei 8.080 de 1990, dispondo sobre suas diretrizes e organização dos serviços de apoio (CONASS, 2009).

A atenção primária a saúde subsidiada pelo SUS, congrega a universalidade, equidade e integralidade do cuidado a todos os grupos populacionais. Frente a isso, tornou-se necessário definir os direitos de cada um deles conforme suas especificidades, sendo que para os idosos a primeira delas foi o Estatuto do Idoso (NAKATANIMACEDO et al., 2015). O Estatuto do Idoso foi aprovado pela Lei no 10.741 de 2003, prevendo em seu conteúdo os direitos mínimos aos idosos, bem como promovendo a conscientização social de respeito e orientação aos profissionais da saúde sobre a assistência prestada (BRASIL, 2003).

Durante a chamada terceira idade de um indivíduo é comum ocorrer à estagnação de sua qualidade de vida, devido às incapacidades físicas devido ao envelhecimento de seu organismo e o surgimento de doenças crônicas. Para isso, os profissionais devem atuar junto ao idoso e sua família, realizando atividades de educação em saúde, buscando restaurar sua vitalidade (VERAS, 2009). No contexto da educação e de tecnologias, principalmente da informática, o idoso é reinserido como ator social em sua comunidade, trazendo consigo o envelhecimento biológico, característica dinâmica e o conhecimento empírico acumulado durante sua vivência (D’ALENCAR, 2002).

A educação para indivíduos idosos não visa à formação categórica, incitada ao mercado de trabalho e meio social consumista, almeja, portanto, a introspecção do conhecimento no próprio ser humano, demarcando a descentralização do saber no jovem e iniciando a quebra de paradigmas (NETTO, 2001). A generalização do sistema de conhecimento voltado à produção econômica percebe o idoso como um estranho no meio educacional, sendo impossível de conceber sua convivência com tecnologias, muito menos interagindo com elas (PATTO, 2000). 
Sendo assim, o presente estudo objetivou conhecer as novas tendências na área da educação e no uso de tecnologias da informática em idosos do Vale do Taquari/RS, Brasil.

\section{METODOLOGIA}

O presente estudo caracteriza-se como uma pesquisa de campo, exploratória e descritiva, com abordagem qualitativa. A pesquisa foi desenvolvida em 15 dos 37 municípios do Vale do Taquari que compõem a 16 ${ }^{\text {a }}$ Coordenadoria Regional da Saúde do estado do Rio Grande do Sul, sendo eles: Bom Retiro do Sul, Boqueirão do Leão, Capitão, Encantado, Estrela, Fazenda Vila Nova, Forquetinha, Ilópolis, Lajeado, Muçum, Putinga, Santa Clara, São José do Herval, Taquari e Teutônia.

A escolha destes municípios foi devido aos dados divulgados em 2011 pela Confederação Nacional de Municípios (CNM). Segundo o órgão, estas comunidades apresentam aproximadamente 329.000 habitantes, sendo reconhecidos 39.117 idosos, ou seja, $12,47 \%$ da população total. Os sujeitos da pesquisa foram 75 idosos residentes nos municípios citados, sendo os critérios de inclusão para a amostra: Ser idoso acima de 60 anos e participar de grupos de convivência, sendo excluídos os que não tinham condições de responder aos questionários.

A coleta dos dados teve sua realização nos espaços de realização de Grupos de Convivência para idosos, e ocorreu mediante contato prévio e aprovação de um representante legal do grupo. As informações foram coletadas por meio de entrevistas ao público alvo, sendo aplicados questionários de autoria do pesquisador, contendo perguntas abertas e fechadas.

As entrevistas tiveram uma duração média de 30 minutos, sendo esclarecidos os objetivos da pesquisa e assegurado aos participantes o direito de interromper seu relato a qualquer momento sem penalizações ou prejuízos para ambas as partes. Todos os participantes assinaram o Termo de Consentimento Livre e Esclarecido (TCLE).

A identidade dos entrevistados foi preservada utilizando-se o nome de flores como codinomes. As entrevistas tiveram seus áudios gravados, transcritos e posteriormente analisados e categorizados conforme prevê a Análise de Conteúdo de Bardin (2011).

Durante todo o processo de desenvolvimento da pesquisa, foram observados e respeitados os aspectos éticos, conforme Resolução No 466, de 12 de dezembro de 2012, do Conselho Nacional de Saúde (CNS), que determina as diretrizes e normas reguladoras de estudos que envolvem seres humanos. Para a realização do estudo, os pesquisadores obtiveram aprovação do projeto pelo Comitê de Ética em Pesquisa (COEP) do Centro Universitário Univates através da CAAE nº 11992112.8.0000.5310. 


\section{RESULTADOS E DISCUSSÃO}

\section{Caracterizações dos sujeitos da pesquisa}

Quanto à caracterização da faixa etária dos sujeitos da pesquisa, verificou-se que dos 75 entrevistados, as idades ficaram entre os 60 a 89 anos. Quanto ao sexo, entrevistaram-se 52 indivíduos do sexo feminino e 23 do sexo masculino.

A partir das informações coletadas nas entrevistas realizadas com os idosos, estas foram organizadas e categorizadas, agrupando-se as respostas que se interrelacionavam, conforme seus conteúdos, resultando em quatro categorias temáticas: (1) O idoso no contexto educacional e político; (2) O ensino de tecnologias da informática como meio de comunicação para o idoso; e (3) As dificuldades econômicas e o acesso à educação e tecnologia.

\section{O idoso no contexto educacional e político}

O idoso no contexto político e educacional obteve proteção de seus direitos há pouco tempo, com a aprovação do Estatuto do Idoso, que realiza a proteção de todos os indivíduos acima de 60 anos de idade, considerado idoso no Brasil (BRASIL, 2003). Conforme verificado através das visitas a campo para realização das entrevistas, a maioria dos idosos carecem de apoio educacional no ensino escolar básico, previsto constitucionalmente.

Os idosos entrevistados relataram serem necessárias aulas para pessoas analfabetas para que consigam assinar seu próprio nome e cuidar de seus depósitos bancários, pois são alvo fácil de meliantes atentos a sua desinformação. No entanto, os idosos não solicitam cursos educacionais de longa duração, pois se consideram incapazes de realizar e desnecessário, visto a necessidade de um ensino de nível básico, conforme as falas:

"Continuar a estudar, para poder mais atenta, conta bancaria" (Copo de Leite).

"Gostaria de estudar para assinar meu nome, e acompanhar a evolução do mundo" (Cravina).

"Sou analfabeto e gostaria de aprender, aprender" (Cravo).

Atualmente as maiores taxas de analfabetismo podem ser evidenciadas na faixa etária acima dos 60 anos de idade, situações estas que associadas coloca a população idosa em risco social, incitando os profissionais a buscarem alternativas para suprir as demandas existentes (SOARES; ISTOE, 2015). O analfabetismo em qualquer faixa etária gera exclusão social e dificuldades em interagir com tecnologias essenciais 
para a sociedade do século XXI, neste caso, a idade avançada constitui-se como fator duplicador de negligencia educacional (FAGUNDES; SANTOS, 2015).

No contexto político da educação, os entrevistados relatam a falta de oportunidades para realização de encontros e grupos de cunho educacional, para suprir suas necessidades. Segundo os idosos as esferas governamentais deveriam realizar ações que contemplassem a população idosa em âmbito local, pois compreendem que políticas a nível nacional não condizem com as realidades em que se encontram, conforme as falas a seguir:

"Mais apoio dos governantes à educação" (Glória da Manhã).

"A familia entender as necessidades, e a comunidade ajudar, criar grupos, ensinar alguma coisa que precisamos" (Cravo).

"Falta educação que ofereça oportunidades de ocupação aos idosos e dê prazer" (Novelos).

A carência de políticas públicas que incentivem a prática de lazer, cultura e atividades em geral para a terceira idade torna-se uma demanda prioritária em nosso país, mesmo com o Estatuto do Idoso em vigência a educação continua sendo subestimada e negligenciada (MENDONÇA, 2015). A necessidade de políticas de fomento aos idosos começou no final do século XIX, devido à clara inversão da pirâmide etária, demonstrando as fragilidades constitucionais que até então distanciavam a terceira idade dos direitos dos demais grupos populacionais (SANTINHA; MARQUES, 2015).

\section{O ensino de tecnologias da informática como meio de comunicação para o idoso}

Durante a realização das entrevistas percebeu-se que os idosos possuem uma necessidade afetiva de comunicar-se com outras pessoas sendo, na maioria das vezes, familiares e amigos que residem em outros municípios. Neste contexto, o uso das tecnologias da informática ganha destaque, servindo como mediador deste diálogo antes reduzido a breves visitas.

Todavia, a informática, bem como outras tecnologias foram incorporadas ao meio social recentemente e nem sempre os idosos sabem manusear estes aparelhos eletrônicos. Para tanto, os entrevistados relatam a necessidade de oportunizar cursos de curta duração, com intuito de ensinar a prática principalmente na área das tecnologias da informática, evidenciado nas falas:

"O idoso necessita mais atenção e mais cursos de como usar o celular para poder se comunicar" (Chuva de Prata).

"Ah mais curso de usar celular, computador" (Yantia). 


\section{"Um curso de uso do computador para que podemos aprender outras coisas"(Resedá).}

As necessidades educacionais para a terceira idade estão aflorando problemas sociais até então negligenciados, no entanto, se as práticas de ensino ocorrer, mesmo que tardias seu impacto nas vivências de cada indivíduo modifica suas compreensões sobre o meio e seus significados (JANINI; BESSLER; VARGAS, 2015). Devido aos novos formatos familiares e exigências sociais, os idosos permanecem sozinhos nesta fase da vida. Na maioria das vezes, os encontros são raros e as tecnologias auxiliam a modificar este ciclo, neste sentido, os idosos buscam adequar-se às exigências (PEREIRA et al., 2015).

Os entrevistados relatam a dificuldade em comunicar-se com outras pessoas por não dominarem as técnicas no uso de aparelhos como o computador e celular, esta dificuldade distancia os laços familiares e torna o processo de envelhecimento traumatizante. Na maioria das vezes, os idosos não recebem o suporte necessário para assimilar uma nova tecnologia em seu meio cultural que até então era desconhecido, tornando-se dependente de outras pessoas para seu manuseio. Deste modo, quando se encontram sozinhos, o contato com estes aparelhos é evitado, conforme as falas a seguir:

"Seria bem legal fazer curso de computador, para me comunicar com os netos" (Sálvia).

"Curso de informática para se comunicar com parentes mais longe"(Oncidio).

"Casa de convivência com uso do computador, internet, celular, para falar com familiares" (Anis).

O acesso universal e igualitário à educação no Brasil é um fenômeno recente, sendo que grande parte dos idosos de hoje, não são alfabetizados por dificuldades no sistema educacional em vigência no final do século XIX, possuindo carências nas áreas de conhecimentos básicos e principalmente tecnológica (JANINI; BESSLER; VARGAS, 2015). A tendência dos idosos em buscarem conhecimento de novas tecnologias diz respeito a facilidades em sua rotina, desde eletrodomésticos até telecomunicações, com o aumento da idade torna-se difícil realizar as tarefas diárias, bem como viajar com frequência, sendo as tecnologias facilitadoras deste processo (FAGUNDES; SANTOS, 2015).

\section{As dificuldades econômicas e o acesso à educação e tecnologia}

O contexto econômico recebeu destaque nas respostas expostas durante a realização das entrevistas, os idosos relatam possuir dificuldades financeiras devido a custos elevados com a cesta básica e medicação. Alguns indivíduos reconhecem a necessidade de trabalharem para pagar as contas básicas em dia, inclusive barrando aquisições de produtos que oferecem conforto a sua rotina, entre eles os tecnológicos. 
Evidenciou-se uma grande necessidade de realização de cursos, sobre saúde, gastronomia, tecnologias e artesanato, durante as entrevistas os idosos demonstraram muito interesse no ensino de coisas novas. No entanto, a grande maioria assume a dificuldade em custear este tipo de curso, sendo impossível sua realização caso não fossem oferecidos gratuitamente e no município de sua residência, segundo as falas abaixo:

"Fazer cursos rápidos de computador de gastronomia e outros de saúde" (Zinias).

"Fazer cursos rápidos de fazer comida e de internet, mas gratuitos para idosos" (Veigelas).

"Cursos grátis de saúde em geral, artesanato, gastronomia" (Acácia).

Com o aumento da expectativa de vida, os idosos não procuram deter-se a atividades exaustivas, e sim, a práticas que façam sentido em seu meio cultural e transmitam uma essência de vitalidade (MINAYO, 2015). O conhecimento oferece dignidade ao ser humano, no idoso, a educação torna-se um diferencial, no entanto as questões econômicas e falta de apoio governamental dificultam sua adesão (JANINI; BESSLER; VARGAS, 2015).

As dificuldades financeiras esbaram diretamente na disponibilidade de acesso a tecnologias como no caso da internet. Os entrevistados mencionam a necessidade de auxilio para custeio deste tipo de tecnologia, principalmente de comunicação, ao passo que sua aposentadoria não oferece subsidio para tal despesa, conforme relato:

"Disponibilidade cursos de informática, internet de graça" (Caladium).

"Ajuda para computador, internet e do celular" (Tuia).

"Sinal de graça para internet iria ajudar muito as pessoas" (Açucena).

A capacitação dos indivíduos na terceira idade para o uso de tecnologias é uma necessidade atual, visto que, para utilizarem os meios de comunicação digitais, necessitam de conhecimentos mínimos da área e disponibilidade de recursos para adquirir os aparelhos necessários (SOARES; ISTOE, 2015). As características de busca do conhecimento possuem aspectos culturais e partem de necessidades do indivíduo em utilizar tais recursos, visto a necessidade de comunicar-se e sentir-se ativo socialmente (SILVA et al., 2015).

\section{CONSIDERAÇÕES FINAIS}

A partir da realização deste estudo demonstraram-se as necessidades de educação e uso de tecnologias pelas pessoas idosas do Vale do Taquari/RS. Buscouse contextualizar o idoso dentro arcabouço político constitucional e verificou-se a sua necessidade de apoio nas questões educacionais, principalmente na disponibilização de modelos educacionais tecnológicos na área da informática para este público. 
Constatou-se a importância e incentivo no uso de tecnologias da informática pelos idosos com a finalidade de auxiliar na sua rotina diária e na comunicação intrafamiliar, importante nesta fase vital. No entanto, verificou-se que as dificuldades econômicas impossibilitam a aquisição de tecnologias, bem como o acesso a educação, para tanto as politicas sociais devem ser revistas e implementadas no sentido de auxiliar o idoso.

Concluiu-se que o idoso sente-se capaz de participar de atividades educativas e interagir com tecnologias da informática se possuir uma boa saúde, sendo responsabilidade das esferas governamentais destinarem recursos para as equipes de saúde realizar ações de prevenção, promoção à saúde e reabilitação de doenças. As equipes multiprofissionais possuem o dever de instrumentalizar a comunidade sobre o envelhecimento, capacitando às famílias e responsabilizando os indivíduos nos processos de envelhecimento seguro e saudável, visto a necessidade tecnológica de educação com a presença da informática.

\section{REFERÊNCIAS}

BARDIN, L. Análise de conteúdo. 70. ed. Lisboa/Portugal, LDA, 2011.

BRASIL. Lei No 10.741 - Dispõe sobre o Estatuto do Idoso e dá outras providências.

Brasília, DF: 2003. Disponível em: http://www.planalto.gov.br/ccivil_03/leis/2003/11 0.741. htm. Acesso em 2016 mar 06.

CNM. Confederação Nacional de Municípios. Disponível em: http://www.cnm.org.br/ dado_geral/mumain.asp?iIdMun=100143425. Acesso em 2016 mar o6.

CONASS. Conselho Nacional de Secretários de Saúde. As Conferências Nacionais de Saúde: evolução e perspectivas. Brasília, 2009.

COSTA, A. E. K. et al. A percepção da equipe de enfermagem acerca do atendimento prestado ao idoso hospitalizado com dor. Caderno Pedagógico, Lajeado, v. 12, n. 3, p. 38-51, 2015.

D'ALENCAR, R. S. "Ensinar a viver, ensinar a envelhecer: desafios para a educação de idosos." Estudos Interdisciplinares sobre o Envelhecimento. v. 4, 2002.

FAGUNDES, V. H.; SANTOS, A.S. As tecnologias de interação e as relações de uso pela terceira idade: um estudo de caso no segmento de linha branca. Blucher Design Proceedings v. 2, n. 2, p. 1251-1261, 2015.

INSTITUTO BRASILEIRO DE GEOGRRAFIA E ESTATÍSTCIA (IBGE). Projeção da população do Brasil por sexo e idade - 1980-2050. Instituto Brasileiro de Geografia e Estatística. 2008.

JANINI, J. P.; BESSLER, D. ; VARGAS, A. B. Educação em saúde e promoção da saúde: impacto na qualidade de vida do idoso. Saúde debate, v. 39, n. 105, p. 480-490, 2015. 
MARIN, M. J. S.; PANES, V. C. B. "ENVELHECIMENTO DA POPULAÇÃO E AS POLÍTICAS PÚBLICAS DE SAÚDE.” Revista do Instituto de Políticas Públicas de Marília, v. 1, n. 1, 2015.

MENDONÇA, J. M. B. Políticas públicas para idosos no Brasil: análise à luz da influência das normativas internacionais. 2015.

MINAYO, M. C. S. Aumento acelerado da expectativa de vida e o desafio de cuidar das pessoas idosas dependentes. Rev. Investigaciones Andina, v.17, . n. 31 p. 170, 2015.

NAKATANI-MACEDO, C. D. et al. "Envelhecimento da População Paranaense: O impacto das Aposentadorias na Renda." Economia \& Região, v. 3, n. 1, p. 27-38, 2015.

NETTO, A. J. Universidade Aberta para a Terceira Idade: uma avaliação crítica. Mundo da Saúde, São Paulo, v. 21, n. 4, jul.-ago., 1997.

PATTO, M. H. S. Mutações do Cativeiro - escritos de Psicologia e Política. São Paulo: Hacker Editores/Edusp, 2000.

PEREIRA, L. V. et al. Intensidade da dor em idosos institucionalizados: comparação entre as escalas numérica e de descritores verbais. Rev. Esc. Enferm, v. 49, n. 5, p. 804-810, 2015.

SANTINHA, G.; MARQUES, S. Ambiente construído, saúde pública e políticas públicas: uma discussão à luz de percepções e experiências de idosos institucionalizados. Saúde e Sociedade, v. 24, n. 3, p. 1047-1060, 2015.

SILVA, E. et al. A legislação brasileira em relação à criança, ao adolescente e ao idoso. Revista Científica da FACERB, v. 2, n.2, p. 96-102, 2015.

SOARES, M. R. P.; ISTO E, R. S. C. Alfabetização e inclusão de pessoas idosas: uma proposta interdisciplinar mediada pelas tecnologias da informação e da comunicação.

LINKSCIENCEPLACE-Revista Científica Interdisciplinar, v. 2, n. 3, 2015.

VERAS, R. Envelhecimento populacional contemporâneo: demandas, desafios e inovações.

Rev Saúde Públic, v. 43, n..3, p. 548- 554, 2009. 